\title{
Very high frequency gravitational wave background in the universe
}

\author{
G.S. Bisnovatyi-Kogan $†$ and V.N.Rudenko $\ddagger$ \\ $\dagger$ Space Research Institute, Moscow, Russia, Profsoyuznaya 84/32, Moscow 117810, \\ Russia and Joint Institute of Nuclear Researches, Dubna, Russia \\ $\ddagger$ Sternberg Astronomical Institute,MSU, Universitetski pr. 13, Moscow, 119899 \\ Russia \\ E-mail: gkogan@mx.iki.rssi.ru
}

\begin{abstract}
.
Astrophysical sources of high frequency gravitational radiation are considered in association with a new interest to very sensitive HFGW receivers required for the laboratory GW Hertz experiment. A special attention is paid to the phenomenon of primordial black holes evaporation. They act like black body to all kinds of radiation, including gravitons, and, therefore, emit an equilibrium spectrum of gravitons during its evaporation. Limit on the density of high frequency gravitons in the Universe is obtained, and possibilities of their detection are briefly discussed.
\end{abstract}

\section{Introduction}

Search for gravitational waves radiated by extraterrestrial sources is carried out now by large experimental teams of different countries. Cryogenic GW bars detectors are in a permanent duty cycle of observations (Allen et al., 2000; Astone et al., 2001), and first test series of observation were performed recently on large gravitational interferometers (Abbott et al., 2003; Shoemaker, 2003). A lot of experimental and theoretical studies have been done in the process of development of this branch of physics conventionally called "Gravitational Wave Experiment". Detailed calculations had been done of continuous and pulse GW-signals emitted by different relativistic objects in the Universe, and algorithms had been developed by many authors (see for example the recent paper of Cutler and Thorne, 2002) of optimal data processing for filtering these signals from the detector's noise background. Modern programs for GW search with both ground based and space apparatus deal with frequency range which does not exceed a few kilocycles per second. The upper frequency limit for relativistic sources is determined by the inverse time of fly-by a strong field region $\nu \leq\left(c / r_{g}\right) \sim 30\left(3 M_{\odot} / M\right) k H z$. The lower frequency, the more intensive GW bursts one could expect according to the modern astrophysical forecast; by in other words massive relativistic stars $M>3 M_{\odot}$ are "too heavy " and "too inertial" for the production a powerful high frequency radiation. Nevertheless a new attention was recently attracted just to the region of very high 
frequency gravitational waves $10^{10}-10^{15} \mathrm{~Hz}$ in connection with the old problem of the possibility of a laboratory GW Hertz experiment (HFGW ,2003; Rudenko, 2003). A stimulating idea is based on the understanding that in the lab one could organize a coherent radiation from a large number of elementary quadruples (atoms or molecules of properly selected media, vortexes in a superconductors etc.). The radiated power is expected to be large enough because the very small factor of the quadruple formula $G / c^{5}$ can be compensated by other factors: the enormous number of participated quadruples up to $10^{22}-10^{24}$ per $\mathrm{cm}^{3}$, sixth power of the oscillating frequency $\omega^{6}$, and a very sharp beaming of the output signal (Rudenko, 2003). In turn an elementary quadruple as a GW detector at very high frequencies should be more effective because its size can be matched to the gravitational wave length (at low frequencies bar detectors and even interferometers have the loose factor (dismatch) $l / \lambda_{g} \ll 1$ ).

Preliminary calculations (Pisarev, 1979; Akihhin, et al. 1985) had shown that at least a couple "GW generator-receiver" might be thought more or less realistic in the lab conditions at "optical frequences" $\nu \sim 10^{14}-10^{15} \mathrm{~Hz}$ where the generated GW power achieves $\sim 1 \mathrm{erg} / \mathrm{sec}$. From the applied and engineering point of view the practical realization of the GW Hertz experiment would open a way to GW communication systems and other applications (HFGW, 2003). In this paper, however, we follow the traditional approach in which the cosmic gravitational radiation is considered as a new channel of astrophysical information. So our goal here is to select conceivable sources of HFGW in astrophysics and to discuss the possibility of their2 detection.

\section{The nature of HFGW in astrophysics}

There are not too many papers in scientific literature with a detailed analysis of astrophysical objects and processes resulted in gravitational radiation at very high frequencies including "optical" and "gamma ray" frequency ranges. A list of HFGW sources theoretically studied more or less up to now is restricted by four different classes of objects: a thermal gravitational radiation of stars, mutual conversion of electromagnetic and gravitational waves in a magnetized interstellar plasma, relic cosmological gravitational wave background and gravitational radiation from very low mass primordial black holes. Below we consider the first three sources very briefly, and the last one in more details.

i) Thermal gravitational radiation of stars.

Thermal motion of charged particles in the stellar high temperature proton-electron plasma produces a gravitation radiation noise. Estimation by the order of magnitude had been performed, for example, in papers of Braginskii and Rudenko (1963), Weinberg (1972), Galtsov and Gratz (1974). One has to calculate more or less rigorously the output of gravitational radiation associated with the elementary process of scattering of two particles (approaching and flying away), and then to make summation over all events at given concentration and velocity distribution of particles.

The most probable frequency of radiation is determined by the frequency of 
collisions $\nu_{c} \sim e^{4} n_{e}(k T)^{-3 / 2} m_{e}^{-1 / 2} \sim 10^{15} \mathrm{~Hz}$, and the highest frequency corresponds to the thermal limit $\omega_{m}=k T / \hbar \sim 10^{18} \mathrm{~Hz}$ (we use here the plasma parameters in the center of the Sun: the electron concentration $n_{e}=3 \cdot 10^{25} \mathrm{~cm}^{-3}$, temperature $T=10^{7} \mathrm{~K}$, here $e, m_{e}$ are the electron charge and mass respectively, and $\hbar$ is the Planck constant). The crude estimate of total "thermal GW power" can be written as (Weinberg 1972)

$$
P_{g} \sim\left(64 G / c^{5}\right) n_{e}^{2} e^{4}\left(k T / m_{e}\right)^{1 / 2} \omega_{m} V_{\odot} \sim 10^{14}-10^{15} \mathrm{erg} / \mathrm{s} .
$$

For the numerical estimate we use the volume of stellar core $V_{\odot}=10^{31}-10^{32} \mathrm{~cm}^{3}$. The result (II) forecasts that at the Earth the "thermal gravitational noise of the Sun" provides a stochastic flux at "optical frequencies" of the order $\sim\left(10^{-13}-10^{-14}\right)$ $\mathrm{erg} / \mathrm{sm}^{2} / \mathrm{s}$.

In the paper of Galtsov (1975) [17] it was calculated that at more high frequencies $\nu \geq 10^{17} \mathrm{~Hz}$ (the x-ray range) the expected GW output might be larger at two orders of value due to the "photocoulomb processes", i.e. the scattering of photons at charged particles with the birth of gravitons.

It is interesting to note that such a level of GW noise is comparable with the GW energy flux from binary stars of the Galaxy at very low frequencies: so the WU Ma (period $0.33 \mathrm{~d}$, distance $110 \mathrm{pc}$ ) provides $\sim 3 \cdot 10^{-13} \mathrm{erg} / \mathrm{cm}^{2} / \mathrm{s}$ and WZ Sge (period $81 \mathrm{~min}$, distance $100 \mathrm{pc}$ ) gives $\sim 4 \cdot 10^{-13} \mathrm{erg} / \mathrm{cm}^{2} / \mathrm{s}$. According to calculation of Mironovskii (1965) the integral radiation of all Galaxy binaries have a stochastic character with a peak at period $\sim 4$ hours, corresponding to the flux $10^{-7}-10^{-8} \mathrm{erg} / \mathrm{cm}^{2} / \mathrm{s}$. Similar accumulation has to take place for the "thermal gravitational noise of stars" but due to large distances to stars the integral radiation cannot exceed the Solar effect. By the order of magnitude the integral gravitational flux from galactic stars at "optical region" might achieve only $10^{-16} \mathrm{erg} / \mathrm{cm}^{2} / \mathrm{s}$ (for an average distance of $100 \mathrm{pc}$, and $\mathrm{N}=10^{9}$ ), although possible effects of close local anomalies in star space distribution has to be addressed.

ii) Graser beams in interstellar plasma.

Possibility of coherent generation of HFGW by electromagnetic waves propagating through an interstellar plasma and magnetic fields initially was considered in the pioneering papers of Gertsenshtein (1962) and Zeldovich (1973). After that many theoreticians had analyzed details of this process in lab and astrophysics. As a last development one could look at the paper of Servin and Brodin (2003), and reference therein, complete theoretical calculations may be found in the book of Galtsov et al. (1984).

Transformations between gravitational and electromagnetic waves is impossible in empty (flat) space but takes place in the presence of ionized media, magnetic fields (or curvature). It creates condition for a parametric interaction these waves with an important sequence of effective energy exchange.

Under special matching conditions ("wave synchronization" or "equality of phase velocities") EM waves generate the GW beam which is amplified by the coherent quadruple oscillations of atoms (as well as of electrons or molecules) of the media 
driven by the EM wave. If a coherent length is long enough the radiated and sharply beamed GW power might achieve a remarkable level. In particular, a propagation of the powerful EM wave in a plasma cylindrical "waveguide" $(r, l ; l \gg r)$ will produce a double frequency GW wave at the Cherenkov angle $\Theta \sim \arccos \left(v_{p} / c\right)$ (where $v_{p}$-is the phase velocity of the EM wave along the "waveguide"). The upper limit of the generated GW power was calculated by Galtsov et. al. (1984). For a relativistic plasma with the characteristic parameter $\gamma=(e E / m c \omega) \sim 1$ which might be produced by very intensive EM optical flux $F \sim 10^{19} \mathrm{~W} / \mathrm{cm}^{2}$ sec (the corresponding field amplitude $E \sim 10^{10} \mathrm{~V} / \mathrm{cm}$ ) the output GW power per unit plasma volume is estimated as

$$
P_{g} \sim \frac{G}{c} \gamma^{2}\left(m_{e} \omega n_{e}\right)^{2}\left(r^{2} l\right)(\lambda / r)^{2} \sim 10^{-5} \mathrm{erg} / \mathrm{cm}^{3} s
$$

the following parameters were used above for the numerical estimate: $\omega=10^{15} \mathrm{rad} / \mathrm{sec}, \lambda=10^{-4} \mathrm{~cm}, n_{e}=10^{22} \mathrm{~cm}^{-3}, r \sim l \sim 1 \mathrm{~cm}$.

In astrophysics such condition might exist in the envelope of supernova or hypernova (gamma-ray sources), jets from accreting dense objects, in the vicinity of neutron stars etc. In fact this type of GW generators at "optical frequencies" is similar to a well known phenomena of EM "cosmic masers" at $\mathrm{GHz}$ and $\mathrm{THz}$ region (Bochkarev 1992), and may be called as "gravitational masers" or "grasers". If the length of the "coherent plasma waveguide" is comparable with the scale of cells of radio masers $\sim 10^{16}-10^{17}$ $\mathrm{cm}$ (Bochkarev 1992), the power of the generated GW beam could achieve $10^{11}-10^{12}$ $\mathrm{erg} / \mathrm{s}$, together with a sharp beaming. A search for such GW long living or transient sources might be carried out with detectors operating in a manner of "reverse scheme" when in a nonlinear "opto-acoustical medium" an incoming HFGW produces a weak electromagnetic wave responce (Akishin et al.,1985; Galtsov et al., 1984).

iii)Relic gravitational wave background

In the frame of the standard cosmological model, the relic stochastic gravitational wave background must exist at present as a result of amplification of initial GW zero quantum fluctuation by variable gravitational field of the expanding Universe (Grishchuk, 1988, 2001, 2003). Such a background exists also in the inflationary and cosmic string cosmologies with some differences concerning the forecasted intensity and spectral features (Allen, 1997). All theories give a non-thermal energy density spectrum for the GW relic background with a growth at low frequencies and a fall in the high frequency region. It reflects a specific parametric mechanism of the GW amplification. Gravitons with wavelengths of the order of the scale factor get a more effective pump from the gravity field of the expanding world.

The spectrum at high frequencies has a cutoff approximately in the region $\nu_{c} \sim 10^{11}$ $\mathrm{Hz}$; it corresponds to the temperature $0.9 \mathrm{~K}$ which $\mathrm{GW}$ relic background would have in the case of the adiabatic evolution (Allen, 1997). Beyond $\nu_{c}$ the spectrum falls down very quickly (some cosmic strings theories give the cutoff shifted to $10^{13}-10^{14} \mathrm{~Hz}$ ). So the "optical" or "gamma-ray" gravitons practically has to be absent in the relict background. In its "radio frequency" part the parametrical GW energy density spectrum follows approximately the law $\Omega_{g w} \sim h^{2}\left(\nu / \nu_{H}\right)^{2}$, where as usual $h$ is the metric 
perturbation, $\Omega_{g w}=\rho / \rho_{c}$ is the normalized GW energy density, $\nu_{H} \simeq 10^{-18}$ is the Hubble frequency. At the cutoff frequency $10^{11} \mathrm{~Hz}$ the intensity estimation is $h \sim 10^{-32}$ for the metric perturbation and $F_{g w} \sim 10^{-5}-10^{-6} \mathrm{erg} / \mathrm{cm}^{2} / \mathrm{s}$ for the $\mathrm{GW}$ flux. These data are very small in comparison with the sensitivity typical of modern gravitational detectors $h \sim 10^{-24}-10^{-25}$ at the frequency range $\left(10^{2}-10^{3}\right) \mathrm{Hz}$. In this region there is the only hope of registering the relict background with an advanced version of $\mathrm{GW}$ interferometers (Grishchuk et al. 2001, Allen, 1997).

iiii) GW radiation from primordial black holes.

The high frequency GW $\nu \geq 10^{10} \mathrm{~Hz}$ could be radiated by relativistic objects with a small mass and gravitational radius $r_{g} \leq 3 \mathrm{~cm}$, i.e. it might be black holes with $M \leq 10^{-5} M_{\odot}$. It was supposed such black holes might exist as a primordial dark matter created at the very early Universe (Carr, 1976; Zeldovich 1980). In the frame of this hypothesis one could speculate over an evolutionary scenario of these objects associated with GW radiation. It could be done for example in the manner of the paper of Nakamura et al.(1997), where the authors supposed an existence binaries composed by MACHO objects, primordial black holes with $M \sim 0.5 M_{\odot}$, and then calculated GW radiation from the coalescence of such binaries. As a part of dark matter these objects may be located in the close environment of the Galaxy increasing the number of expected coalescences per year. The estimations of Nakamura et al. (1997) gave the probability $510^{-2}$ events/year/gal, which is at least two order of magnitude larger than for conventional relativistic binaries (Cutler and Thorne 2002). A hypothesis of binaries composed by mini black holes with much smaller masses (so called "holeums") was considered recently by Chavda and Chavda, 2002).

However there is an obvious mechanism for HFGW production based on the effect of evaporation of black holes of small masses through the "graviton degree of freedom". In the rest part of this paper we will try to estimate an efficiency of this channel which might compensate the deficit of high frequency gravitons in the relic gravitational wave background. At first we remind briefly the main features of the effect.

It was shown by Hawking (1975), following a thermodynamic analysis of Bekenstein (1973), that black hole has properties of a black body with a temperature $T_{\mathrm{H}}$.

$$
T_{\mathrm{H}}=\frac{\hbar c^{3}}{8 \pi k G M_{\mathrm{bh}}}=6.2 \cdot 10^{-8}\left(\frac{M_{\odot}}{M_{\mathrm{bh}}}\right) K .
$$

The temperature $T_{\mathrm{H}}$ may be estimated phenomenologically from the obvious assumption, that the characteristic length of the emitting radiation is of the order of the gravitational radius of the black hole. Assume that the wave is not absorbed when

one quarter of its length exceeds the gravitational radius, so that $\lambda_{\text {lim }}=\frac{8 G M_{\mathrm{bh}}}{c^{2}}$. The corresponding frequency and quantum of energy are

$$
\nu_{l i m}=\frac{c}{\lambda_{l i m}}=\frac{c^{3}}{8 G M_{\mathrm{bh}}}, \quad \varepsilon_{l i m}=2 \pi \hbar \nu=\frac{\pi}{4} \frac{\hbar c^{3}}{G M_{\mathrm{bh}}} .
$$


The black body may not absorb quanta with wavelength $\lambda>\lambda_{\text {lim }}$, so it may emit them, see also Kuchiev (2003). The black body emits the planckian spectrum of radiation, whose maximum energy $\varepsilon_{m}$ is connected with the temperature $T$ as $\varepsilon_{m}=2.822 \mathrm{kT}$ (Landau and Lifshitz, 1995). While almost all quanta with $\varepsilon<\varepsilon_{\text {lim }}$ are emitted by the black body, the limiting energy should be much greater than the energy of the maximum $\varepsilon_{m}, \varepsilon_{\text {lim }}=\eta \varepsilon_{m}, T=\frac{\varepsilon_{\text {lim }}}{2.822 \eta k}$, with $\eta \gg 1$. Taking $\eta=\frac{2 \pi^{2}}{2.822}=7.0$, we come to the Hawking temperature (3) .

\section{Energy spectra of emitting particles}

The black body spectrum of the emitting gravitons is described by the Planck formula (Landau and Lifshitz, 1995). Like photons, the massless gravitons have two independent polarizations, and therefore have the same statistical weight $g_{\mathrm{gr}}=g_{\mathrm{ph}}=2$, in spite of twice larger spin $s_{\mathrm{gr}}=2, s_{\mathrm{ph}}=1$. The radiation of the black hole at low frequencies $h \nu \ll k T_{\mathrm{H}}$ is overestimated by the Planck formula, but we will ignore this.

$$
\frac{d E_{\mathrm{gr}, \nu}}{d \nu}=\frac{g_{\mathrm{gr}} h^{4}}{2 \pi^{2} \hbar^{3} c^{3}} \frac{\nu^{3}}{e^{h \nu / k T}-1} \mathrm{erg} / \mathrm{cm}^{3} / \mathrm{Hz} .
$$

The spectral density $F_{\mathrm{gr}, \nu}$ of the flux of gravitons from the unit surface of the black hole is

$$
F_{\mathrm{gr}, \nu}=\frac{c}{4} \frac{d E_{\mathrm{gr}, \nu}}{d \nu} \mathrm{erg} / \mathrm{sm}^{2} / \mathrm{s} / \mathrm{Hz}
$$

The total equilibrium energy density $E_{\text {gr }}$ and flux $F_{\text {gr }}$ of gravitons is obtained after the integration over the frequency

$$
E_{\mathrm{gr}}=\frac{2 g_{\mathrm{gr}}}{c} \sigma T^{4}, \quad F_{\mathrm{gr}}=\frac{g_{\mathrm{gr}}}{2} \sigma T^{4}, \quad \sigma=\frac{\pi^{2} k^{4}}{60 \hbar^{3} c^{2}} .
$$

Decrease of the mass of a black hole due to its evaporation and emission of massless or ultrarelativistic particles is written as

$$
\frac{d M_{\mathrm{bh}}}{d t}=-\left(\Sigma_{\mathrm{boz}} g_{i}+\frac{7}{8} \Sigma_{\mathrm{fer}} g_{i}\right) \frac{\sigma T_{\mathrm{H}}^{4}}{2 c^{2}} 4 \pi\left(\frac{2 G M_{\mathrm{bh}}}{c^{2}}\right)^{2},
$$

where the sums $(\Sigma)$ are taken over the statistical weights of all kinds of the particles, bozons and fermions. For the sum of photons, gravitons, 3 types of massless neutrino (antineutrino) and their massive leptons, we have, using (3), and the equality $g_{\mathrm{ph}}+g_{\mathrm{gr}}+$ $3 \frac{7}{8}\left(g_{\nu}+g_{\tilde{\nu}}+g_{\mathrm{e}^{-}}+g_{e^{+}}\right)=2+2+3 \frac{7}{8}(1+1+2+2)=\frac{79}{4}$,

$$
\frac{d M_{\mathrm{bh}}}{d t}=-158 \pi \frac{G^{2} M_{\mathrm{bh}}^{2}}{c^{6}} \sigma T_{\mathrm{H}}^{4}=-\frac{79}{8^{4} 30 \pi} \frac{\hbar c^{4}}{G^{2} M_{\mathrm{bh}}^{2}} .
$$

Solving (8) we find a time $t_{\mathrm{bh}}$ of the black hole evaporation. The main portion of the energy, and main evaporation time is collected at initial stages. We take (8) for all masses, while at $k T_{\mathrm{H}}>\sim 0.3 m_{\pi} c^{2} \approx 100 \mathrm{MeV}$ pions started to be radiated, and at 
larger $T_{\mathrm{H}}$ other massive particles, up to quarks and gluons are emitted (Barrau et al., 2003).

$$
t_{\mathrm{bh}}=\frac{8^{4} 10 \pi}{79} \frac{G^{2} M_{0}^{3}}{\hbar c^{4}}=8.50 \cdot 10^{18} M_{15}^{3}, \quad M_{15}=\frac{M_{\mathrm{bh}}}{10^{15} \mathrm{~g}} .
$$

Black holes evaporate during the cosmological time $5 \cdot 10^{17} \mathrm{~s}$ when its initial mass $M_{0}$ is less than $M_{b}=4 \cdot 10^{14} \mathrm{~g}$. (Page, 1976; Novikov and Frolov, 1998). Its temperature then exceeds

$$
T_{\mathrm{H}}>3.1 \cdot 10^{11} \mathrm{~K}=26.6 \mathrm{MeV} .
$$

We see than that we may neglect pions and barion-antibarion emission at the initial stages of evaporation of black holes with $M \geq M_{b}$. We also have taken ultrarelativistic Fermi distributions for all leptons, overestimating the radiation of $\tau$ - leptons. While we neglect losses, connected with other particles, like pions, we may expect that this approximation gives reasonable result for the black hole evaporation rate.

Let us find the spectrum of the gravitons or photons (bosons) $\mathcal{E}(\nu)$, produced at the evaporation of the black hole with the initial mass $M_{0}$. We integrate (5) over the whole evaporation time, which is reduced to the integration over the mass, using (8). We have

$$
\begin{array}{r}
\mathcal{E}(\nu)=\frac{15}{79 \pi^{5}} \frac{\hbar c^{5}}{G} \frac{h}{(h \nu)^{2}} \int_{0}^{x_{0}} \frac{x^{4} d t}{e^{x}-1} \mathrm{erg} / \mathrm{Hz}, \\
x=\frac{8 \pi G M_{\mathrm{bh}} h \nu}{\hbar c^{3}}, x_{0}=\frac{8 \pi G M_{0} h \nu}{\hbar c^{3}} .
\end{array}
$$

The spectrum (11) has a maximum at

$$
h \nu_{m}=\frac{\hbar c^{3} x_{m b}}{8 \pi G M_{0}}
$$

where $x_{m \mathrm{~b}}$ is a solution of the integral equation

$$
\frac{x_{m \mathrm{~b}}^{5}}{e^{x_{m \mathrm{~b}}}-1}=2 \int_{0}^{x_{m \mathrm{~b}}} \frac{x^{4}}{e^{x}-1} d x, x_{m \mathrm{~b}}=3.933350238 .
$$

In the approximation that the black hole emits the planckian spectrum, the maximum of the radiation spectra of photons and gravitons corresponds to the energy $h \nu_{m}$ and is connected with the Hawking temperature of the initial mass black hole $T_{\mathrm{H} 0}$ as

$$
h \nu_{m}=x_{m \mathrm{~b}} k T_{\mathrm{H} 0}=3.933 k T_{\mathrm{H} 0},
$$

which is not far from the maximum of the Planck spectrum (Landau and Lifshitz, 1995) $\nu_{\mathrm{P} m}$, corresponding to one temperature $T: h \nu_{\mathrm{P} m}=x_{\mathrm{P} m} k T=2.822 k T$. $\ddagger$ It happens because the main energy is emitted when the black hole mass was not far from the initial mass $M_{0}$. For black holes with $M_{0}=4 \cdot 10^{14} \mathrm{~g}$, which are evaporated to the present $\ddagger$ More exactly $x_{\mathrm{P} m}=2.821439372$, and is a root of the equation $3\left[1-\exp \left(-x_{\mathrm{P} m}\right)\right]=x_{\mathrm{P} m}$ 
time, the maximum energy of emitted gravitons (and photons), with account of (10), is about $\varepsilon_{g r, m}=x_{m \mathrm{~b}} k T_{\mathrm{H}}=0.105 \mathrm{GeV}$.

The equilibrium energy spectrum $E(\varepsilon)$ of the massless fermions, or any other ultra-relativistic fermions with a statistical weight $g_{f}=2$ emitted during a black hole evaporation (neutrino+antineutrino, electrons, positrons), is found analogously to (11)

$$
\begin{aligned}
\mathcal{E}(\varepsilon)=\frac{15}{79 \pi^{5}} \frac{\hbar c^{5}}{G} & \frac{h}{\varepsilon^{2}} \int_{0}^{x_{0}} \frac{x^{4} d x}{e^{x}+1} \mathrm{erg} / \mathrm{Hz}, \\
x & =\frac{8 \pi G M_{\mathrm{bh}} \varepsilon}{\hbar c^{3}}, x_{0}=\frac{8 \pi G M_{0} \varepsilon}{\hbar c^{3}} .
\end{aligned}
$$

The spectrum (11) has a maximum at

$$
\varepsilon_{m}=\frac{\hbar c^{3} x_{m \mathrm{f}}}{8 \pi G M_{0}}
$$

where $x_{m \mathrm{f}}$ is a solution of the integral equation

$$
\frac{x_{m \mathrm{f}}^{5}}{e^{x_{m \mathrm{f}}}+1}=2 \int_{0}^{x_{m \mathrm{f}}} \frac{x^{4}}{e^{x}+1} d x, x_{m \mathrm{f}}=4.350853675, \quad \varepsilon_{m}=4.351 k T_{\mathrm{H} 0},
$$

while the maximum of the Fermi spectrum of ultrarelativistic particles with zero chemical potential corresponds to $\varepsilon_{\mathrm{F} m}=x_{\mathrm{F} m} k T=3.13101972 k T$, and $x_{\mathrm{F} m}$ is found from the equation $3\left(1+e^{-x_{\mathrm{F} m}}\right)=x_{\mathrm{F} m}$.

\section{Estimations of the flux of high-energy gravitons}

The restrictions to the density of the primordial black holes, following from the observations of the gamma ray background (Barrau et al., 2003), are $\Omega_{P B H}<$ $3.3 \cdot 10^{-9}, \Omega_{P B H}=\rho_{P B H} / \rho_{c r}$. Taking (Naselskij et al, 2003) the Hubble constant $H=71 \mathrm{~km} / \mathrm{s} / \mathrm{Mpc}$, and $\rho_{c r}=1.8 \cdot 10^{-29} \mathrm{~g} / \mathrm{cm}^{3}$, we obtain the restriction to the energy density of the gravitons emitted by $\mathrm{PBH}$ as

$$
\mathcal{E}_{g r} \approx \frac{8}{79} \rho_{P B H} c^{2}=2.7 \cdot 10^{-18} \mathrm{ergs} / \mathrm{cm}^{3}=1.7 \cdot 10^{-15} \mathrm{GeV} / \mathrm{cm}^{3}
$$

The energy of the gravitons emitted during evaporation of $\mathrm{PBH}$ is about $0.1 \mathrm{GeV}$, so the upper limit to the flux of the $0.1 \mathrm{GeV}$ gravitons is $F_{g r}<c \mathcal{E}_{g r} / 4 \approx 1.3 \cdot 10^{-4}$ gravitons $/ \mathrm{cm}^{2} / \mathrm{s}$.

Black holes with lower initial masses evaporate earlier and emit more energetic particles, but due to the red shift $z$, the observed energy of the evaporated particles at the present time decreases with the initial mass. According to (31), (91), (14) the evaporation time $t_{\mathrm{bh}}$ and co-moving energy of the maximum of the spectrum $E_{m}=h \nu_{m}$ for gravitons and photons are

$$
t_{\mathrm{bh}}=5 \cdot 10^{17}\left(\frac{M}{4 \cdot 10^{14}}\right)^{3} \mathrm{~s}, \quad E_{m}=105\left(\frac{4 \cdot 10^{14}}{M}\right) \mathrm{MeV} .
$$


Let us find the energy of the photon or the graviton, produced at $t=t_{\mathrm{bh}}$ by black holes evaporated at that time, with which it comes to the present time. We take into account, that in the matter dominated universe at zero pressure (after recombination) we have (Peebles, 1971)

$$
P=0, \quad 1+z=\left(\frac{5 \cdot 10^{17}}{t}\right)^{2 / 3}, E_{m 0}=\frac{E_{m}}{1+z}=105\left(\frac{M}{4 \cdot 10^{14}}\right) \mathrm{MeV} .
$$

Before recombination in the radiation dominated universe the following relations are valid at $z>z_{\text {rec }}$ :

$$
P=\frac{\varepsilon}{3}, \frac{1+z}{1+z_{r e c}}=\left(\frac{t_{r e c}}{t}\right)^{1 / 2}, E_{m, r e c}=E_{m} \frac{1+z_{r e c}}{1+z}, \rho=\frac{4.5 \cdot 10^{5}}{t^{2}} \mathrm{~g} / \mathrm{cm}^{3} .
$$

In the universe with two stages of expansion the relations (20) and (21) are valid after and before recombination. Taking the recombination at $z_{\text {rec }}=1000,\left(t_{\text {rec }}=\right.$ $\left.5 \cdot 10^{17} /\left(1+z_{\text {rec }}\right)^{3 / 2}=1.6 \cdot 10^{13} \mathrm{~s}, T_{\text {rec }}=3000 \mathrm{~K}\right)$, we obtain the ratio of the energies at the moment of recombination and at present time $E_{m 0}=10^{-3} E_{r e c}$, and the mass of a black hole evaporated at the moment of recombination is equal to

$$
M_{b h, r e c}=\frac{4 \cdot 10^{14}}{10^{1.5}}=1.3 \cdot 10^{13} \mathrm{~g} .
$$

The formula (20) for the observed energy of evaporated photons (gravitons), in the case of free propagation, is valid in the mass interval $1.3 \cdot 10^{13}<M_{b h}<4 \cdot 10^{14}$. Combining (19) - (22), we obtain the observed energy of freely propagating quanta (gravitons) emitted by black holes evaporated to the time $t<t_{\text {rec }}$ as

$$
E_{m 0}=\frac{105}{\left(1+z_{r e c}\right)^{0.25}}\left(\frac{M}{4 \cdot 10^{14}}\right)^{0.5} \mathrm{MeV}, \quad \text { for } \quad M_{b h}<\frac{4 \cdot 10^{14}}{10^{1.5}} \mathrm{~g} .
$$

It was shown by Zeldovich and Sunyaev (1969) that any extra heat introduced into the universe will be thermalized, provided it is introduced at $z>z_{p h}=10^{6}$. The point $z=z_{p h}=10^{6}$ corresponds to $t=t_{p h}=1.6 \cdot 10^{7} \mathrm{~s}$, in the universe with two stages. The corresponding temperature of the universe is connected only with the redshift during adiabatic expansion of a pure photon gas, and is equal to $T_{p h}=3 \cdot 10^{7} \mathrm{~K}$.

Black holes evaporated at $z>z_{p h}$ do not disturb the Planckian cosmic microwave background (CMB), but produce energetic nonthermal gravitons and neutrino. The heat produced at $z>z_{\nu}$ is so large that neutrino thermalization have time to occur, produce only energetic gravitons. The values $z_{\nu}, t_{\nu}, T_{\nu}$, may be estimated from the condition that the mean free path of neutrino due to interaction with pairs is much smaller than the horizon. The number density of the electron-positron and other pairs may be estimated, using (21), as

$$
N_{e^{+} e^{-}}=\frac{\rho c^{2}}{x_{F m} k T}=\frac{\left(\rho c^{2}\right)^{3 / 4} a^{1 / 4}}{k x_{F m} n^{1 / 4}}=\frac{4.4 \cdot 10^{31}}{t^{3 / 2}} .
$$

In the radiation dominant plasma $T=\left(\rho c^{2} / n a\right)^{1 / 4}, a=7.565 \cdot 10^{-15}$ is a constant of the radiation density, $n$ is the number of sorts of low-mass and massless particles taken as $n=6$. 
Estimating the cross section of neutrino interaction with electrons as $\sigma_{\nu e} \approx 10^{-44}$ $\mathrm{cm}^{2}$, we obtain from the relation $\frac{1}{\sigma_{\nu e} N e^{+} e^{-}} \sim \frac{c t}{10} \ll c t$ the corresponding values as

$$
t_{\nu}=2 \cdot 10^{-6} \mathrm{~s}, \quad z_{\nu}=3 \cdot 10^{12}, \quad T_{\nu}=8 \cdot 10^{12} \mathrm{~K} .
$$

The primordial black holes with masses less than $M_{p h}=1.3 \cdot 10^{11} \mathrm{~g}$, or $M_{\nu}=6 \cdot 10^{6}$ $\mathrm{g}$, are evaporating without disturbing CMB spectrum, or without disturbing also the neutrino background spectrum. The spectra maximum of gravitons emitted at these two moments, according to (3), (14), are equal to $E_{m, p h}=320 \mathrm{GeV}, E_{m, \nu}=7 \cdot 10^{6}$ $\mathrm{GeV}$. Using corresponding redshifts, we obtain the following energies of the gravitons at present time: $E_{m 0, p h}=320 \mathrm{keV}, E_{m 0, \nu}=2 \mathrm{keV}$.

Supposing, that considerable $(\sim 0.5)$ part of the CMB was produced by primordial black holes evaporation, we obtain the upper limit of the flux of gravitons, produced at different redshifts. The CMB radiation corresponds to $\Omega \sim 7 \cdot 10^{-5}$, which exceeds by $2 \cdot 10^{4}$ times the upper limit $\Omega_{P B H}$ for black holes evaporated by the present time. The upper limits for graviton fluxes, produced by black holes evaporated at earlier times, are equal to $\S$

$$
\begin{aligned}
& F_{g r}^{(p h)}<\sim 400 \text { gravitons } / \mathrm{cm}^{2} / \mathrm{s} \text { at } E_{m 0, p h}^{(2)}=320 \mathrm{keV}, \\
& F_{g r}^{(\nu)}<\sim 6 \cdot 10^{4} \text { gravitons } / \mathrm{cm}^{2} / \mathrm{s} \text { at } E_{m 0, \nu}^{(2)}=2 \mathrm{keV} .
\end{aligned}
$$

In the first case similar fluxes of neutrinos with approximately the same energies as gravitons are expected, in the last case neutrinos should be thermalized. According to (23), the gravitons with energies of optical spectrum $(\sim 1 \mathrm{eV})$ may be produced by the black holes with mass $\sim 1$ gramm, and since the upper limit for the total flux of gravitons remains the same, we have

$$
F_{g r}^{(o p t)}<\sim 1.2 \cdot 10^{8} \text { gravitons } / \mathrm{cm}^{2} / \mathrm{s}
$$

On the other hand, the optical photons are produced by presently evaporating black holes with mass about $5 \cdot 10^{-12} M_{\odot}$, corresponding to the Hawking temperature about 1 $\mathrm{eV}$. The energy flux from the evaporation of such black holes with the total mass equal to solar mass and solar surface temperature is

$$
F_{b h}^{(o p t)}=F_{\odot}\left(\frac{R_{b h}}{R_{\odot}}\right)^{2} \frac{M_{\odot}}{M_{b h}}=10^{-22} F_{\odot},
$$

taking into account the relation for the gravitational radius $R_{b h} \equiv r_{g}=\frac{2 G M}{c^{2}}=$ $3 \cdot 10^{5} \mathrm{M} / M_{\odot} \mathrm{cm}$. While we cannot increase the total mass of black holes more than $\sim 50$ times compared to the present total mass of stars, the optical flux of presently evaporated black holes does not exceed $10^{-20}$ of total stellar optical flux, i.e. completely negligible. Roughly speaking, the optical stellar flux is of the order of the flux of the $\S$ It was noted by Novikov et al. (1979), that BH with masses $10^{10}--10^{13} \mathrm{~g}$ evaporated at the epoch of nucleosynthesis influence the helium production, so the upper limit of the graviton flux in the first line of (26) decreases about an order of magnitude. 
microwave background, so the flux of optical gravitons from presently evaporating black holes does not exceed $10^{-20}$ of its upper limit in (27).

The above estimations have been done for pure equilibrium (Planck and Fermi) spectra of evaporating particles. Calculations of Page (1976), where deviation from equilibrium spectra had been taken into account, gave for non-rotating black holes redistribution of the emitting particles in favor of particles with lower spins. The amount of radiated gravitons is about 50 times less than the sum of photons and pairs, which are transformed into photons during thermalization. In the equilibrium the graviton flux is about 3 times less than that of the sum of radiation and pairs. Therefore, the estimations of the graviton fluxes in (26) should be reduced by a factor of 20 . In the case of evaporation of a rapidly rotating black hole the situation is opposite, and radiation of particles with larger spins (gravitons) (Novikov and Frolov, 1998) prevails. In that case we may expect much lower reducing (if any) of the graviton flux estimations in (26).

\section{Perspectives of the HFGW detection}

Detection of very high frequency gravitational radiation of cosmic origin previously did not attract a serious attention and corresponding devices were not developed. One can appeal only to ideas of conceivable lab detectors for the Hertz GW experiment in the ultra high frequency radio range $\left(10^{9}-10^{12}\right) \mathrm{Hz}$ and at optical frequencies $\left(10^{13}-10^{15}\right)$ Hz. There is nothing with respect to the GW detection in the region of "gamma ray" frequencies. However a rough estimate of the "detectable signal" may be given from general principles without a consideration of a concrete technical construction of devices.

A relative energy variation of some HFGW detector $\Delta W / W$ has to be proportional to metric perturbations $h$ accumulated during the measurement time $\tau_{m}$, i.e the "signal response" is expected as $\Delta W / W \sim h \omega \tau_{m}$. For to be registered it must overcome the

quantum fluctuation standard $(1 / \sqrt{N}) \simeq \sqrt{\hbar \omega / W}$, where $N$ is a number of quanta of the electromagnetic pump field of the detector. From this condition one can define a minimal registered $h_{\text {min }}$ and then a limit for the detectable GW energy flux.

Such an approach is valid for the resonance deterministic signals and detectors having not deal with the condition of "wave synchronism", for example it might be electromagnetic or optical resonators of a large volume (Grishchuk, Sazhin 1975). In the case of receiving stochastic signals a difference consists in a more slow rate of accumulation, i.e the accumulation factor is $\sim \sqrt{\omega \tau_{m}}$. For a single act of measurement (one detector, one switch on-off) the value of accumulation factor is limited by the detector relaxation time $\sqrt{\omega \tau_{r}} \simeq \sqrt{Q}$, where $Q$ is the oscillation quality factor. However if there is a possibility to use simultaneously two coherent (synchronized) detectors to separate (to filter) correlated "GW-noise signals" from independent intrinsic noises of detection devices then the accumulation might be continued and the effective response would grow with time as $\sqrt{\tau_{m} / \tau_{r}}$ (see for example (Allen B 1997)) under an assumption of the permanent existence of the measured GW noise background. Finally, the potential 
detection sensitivity may be written as

$$
h_{\text {min }} \sim \sqrt{\frac{\hbar \omega}{W}} \frac{1}{\sqrt{Q}} \sqrt{\frac{\tau_{r}}{\tau_{m}}} \simeq \sqrt{\frac{\hbar}{W \tau_{m}}}
$$

Thus, the principal level of the potential sensitivity is defined only by a total energy stored in a detector and a value of allowed measurement time.

For numerical estimate let's take parameters used in the paper of Grishchuk (2003) for the electromagnetic detector at frequencies $10^{8}-10^{9} \mathrm{~Hz}$. The electromagnetic field $E=10^{4} \mathrm{~V} / \mathrm{cm}$, the detector cell volume $\lambda^{3} \simeq 10^{6} \mathrm{~cm}^{3}$, the number of independent cells $N=1000$, (then the total energy stored $W \simeq(4 / 25) 10^{21} \mathrm{erg}$ ); the measured time $\tau_{m}=10^{7}$ sec (three months).

With these parameters the formula (29) results in the estimate $h_{\min } \sim 10^{-28}$. The only conceivable way for further enhancing the sensitivity may be associated with using of a special quantum squeezed states of electromagnetic field inside the detector with a suppressed variance of photon noise (Grishchuk 2003). However it is unlikely to wait an improvement more a one-two orders of the magnitude i.e. the extremely optimistic estimate of the detection sensitivity can not be prolonged below the $h_{\text {min }}=10^{-30}$. Unfortunately even this level of "delectability" is insufficient to register very high frequency stochastic GW background.

In reality the average amplitude of metric perturbation integrated in the bandwidth $\Delta \omega \sim \omega=E_{m} / \hbar$ is defined by the spectral density of GW flux $F_{\omega}$ according to the following relation (Landau, Lifshitz 1988)

$$
<h>\left.\right|_{\omega} \simeq \sqrt{\frac{16 \pi G}{c^{3} \omega^{2}} F_{\omega} \Delta \omega} \simeq 310^{-19} \omega^{-1}\left(\frac{F_{0}}{1 \mathrm{erg} / \mathrm{sm}^{2} \mathrm{sec}}\right)^{1 / 2}
$$

where we define $F_{0}=F_{\omega} \Delta \omega$.

For primordial gravitons produced by mini black hole evaporation $F_{0} \sim 10^{-4}$ $\mathrm{erg} / \mathrm{cm}^{2}$ sec in the very wide frequency range, (26), (27). Then we have from (30) the estimation $\left\langle h>\left.\right|_{\omega} \sim 310^{-31}\right.$ in the "radio high frequency range" $\omega=10^{10} \mathrm{rad} / \mathrm{sec}$ and much less $<h>\left.\right|_{\omega} \sim 310^{-36}$ at the "optical frequencies" $\omega=10^{15} \mathrm{rad} / \mathrm{sec}$, i.e. $E_{h \nu} \sim(1-10) \mathrm{eV}$. Thus one has to conclude that a direct detection of the "black hole gravitons" is impossible. The same is valid for the high frequency tail of the relict GW background and thermal GW noise of stars.

A high frequency GW source has to provide the flux at least $F_{0} \sim(1-10) \mathrm{erg} / \mathrm{cm}^{2}$ sec at the Earth surface to be detected in principle. A weak hope to get something like this may be associated with GW grasers if they would have an extremely sharp beaming and would be discovered nearly the Earth. Then for their detection one could apply detectors based on the wave synchronism of GW and optical waves in nonlinear opto-acoustical media (Pisarev 1979, Bessonov 1998, Rudenko 2003) which looks more effective but at present is not yet completely developed.

Among the known detectors, the optical GW-interferometers with FP cavity in the arms in principle are able to detect the short wave GW-radiation $\lambda_{g} \ll L$ under a special condition of the so called "grav-optical resonance" $\left(2 L / \lambda_{g}\right)=k$, where $k$ is 
a large integer number (for example: $k=10^{3}, L=10^{5} \mathrm{~cm}, \lambda_{g}=10^{2} \mathrm{~cm}$ ) (Rudenko, Sazhin 1980). This condition means that photons meet the same gravitational wave phase at moments of their reflection from interferometer arm mirrors. It results in the accumulation of optical response defined by the "finess factor" (number of photon round trips during of the optical relaxation time) i.e. it is a similar accumulation effect which characterizes an operation of GW interferometers at low frequencies. So the expected sensitivity can not be better than the advanced sensitivity of the VIRGO and LIGO interferometers or LCGT project (Kuroda et.al.) $10^{-22}-10^{-24} \mathrm{~Hz}^{-1 / 2}$.

\section{Conclusions}

Analysis of high frequency GW produced by evaporation of black holes of very small masses $1-10^{7} \mathrm{~g}$ have shown, that the energy density of such GW may be of the order of the present energy density of optical photon background produced by stars. Production of this GW does not change any characteristics of other backgrounds, because all products of the $\mathrm{BH}$ evaporation except $\mathrm{GW}$ should be thermalized. In the case of $\mathrm{BH}$ of larger masses $10^{7}-10^{11} \mathrm{~g}$ we may expect, in addition to $\mathrm{GW}$, the formation of neutrino background with energies in the X-ray region $2-300 \mathrm{keV}$ for both types of the background. Note that considerably larger energy density of the neutrino background at energies in $\mathrm{MeV}$ region is produced by core-collapse supernovae during formation of neutron stars (Bisnovatyi-Kogan and Seidov, 1982).

The present status of experimental technique does not permit us to expect the detection of high energy GW stochastic background in the nearest future, but it should be beared in mind for further development. Detection of the such GW, whose origin is definitely could be connected with primordial $\mathrm{BH}$ evaporation, would give an answer to fundamental questions of the physics of $\mathrm{BH}$ evaporation, the origin of cosmic microwave background, and physical processes in the early universe.

Authors would like gratitude colleagues from MSU prof. Lipunov V.M. and prof. Postnov K.A. for useful discussions; prof Galtsov D.V. for comments concerning the generation of HFGW by the relativistic plasma.

The paper was partly supported from RFBR grant 02-02-16900 and INTAS grant 00491.

[1] Abbott B. et.al. 2003, gr-qc/038069, 21 Aug.

[2] Akishin P G, Bogolyubov P N, Pisarev A F 1985, preprint JINR N13-85-968 (in Russian)

[3] Allen B.1997, in Relativistic Gravitation and Gravitational Radiation, p.373, ed. Marck J.A., Lasota J.P., Cambridge Univ. Press.

[4] Allen Z.A. et al. 2000, Phys.Rev.Lett.85, 5056

[5] Astone et al. 2001, Class.Quantum Grav., 18, 243

[6] Barrau A, Boudoul G, Derome L. 2003, astro-ph/0304528.

[7] Bekenstein J.D. 1973, Phys. Rev. D, 7, 2333.

[8] Bessonov E.G. 1998, physics/980237,v2, 27 Feb.

[9] Bisnovatyi-Kogan G. S., Seidov Z. F. 1982, Astron. Zh. 59, 213 (Sov. Astron. 1982, 26, 132).

[10] Bochkarev N.G.1992, Fundamentals of physics of interstellar media, ed. Moscow University (in Russian) 
Very high frequency...

[11] Braginskii V.B., Rudenko V.N. 1963, Scientific notes of Kazan University, v.123, n 12, p.96-108, ed. Kazan University 1963 (in Russian).

[12] Carr B. 1976, ApJ, 206, 8.

[13] Chavda A.L., Chavda L.K. 2002, GRG 19, 2927. (gr- qc/038054, 2003), 17 Aug 2003)

[14] Cutler C.,Thorne K.S. gr-qc/0204090 v1 30 Apr 2002 (in General Relativity and Gravitation, ed. Bishop N.T., Maharaj S.D., p. 72, World Scientific 2002)

[15] Galtsov D.V., Gratz Yu.V., Petukhov V.I. 1984, Radiation of gravitational waves by electrodynamic systems, ed. Moscow University (in Russian)

[16] Galtsov D.V., Gratz Yu.V. 1974, Sov. Phys. Journ.,17, n 12, p.94

[17] Galtsov D.V. 1975, Sov. Phys. JETP, 40, p.211

[18] Gertsenshtein M.E, 1962, Sov. Phys. JETP, 14, 84.

[19] Grishchuk L.P.1988, Uspekhi Fizicheskih Nauk, 156, 297. (in Russian)

[20] Grishchuk L.P., Lipunov V.M., Postnov K.A. et. al., 2001, Uspekhi Fizicheskih Nauk, 171, 4. (in Russian)

[21] Grishchuk L.P., Sazhin M.V. 1975, Sov.Phys.JETP, 41,787.

[22] Grishchuk L.P., 2003, gr-cq/03005051, 13 May .

[23] Hawking S.W. 1975, Commun. Math. Phys. 43, 199.

[24] HFGW conference, McLean, May 2003, http: groups.yaho.com/group/SpaceWork/907; www.gravwave.com

[25] Kuchiev M. Yu. 2003, Reflection, radiation and interference for black holes. gr-qc/0310051

[26] Kuroda K., Ohashi M., Myoki S. et. al. 1999, Int.J.Mod.Phys. 8, n.5, 557-9.

[27] Landau L.D., Lifshitz E.M. 1995, v.5 Statistical Physics. Nauka. Moscow.

[28] Landau L.D., Lifshitz E.M. 1988, v.2 The classical theory of fields. Nauka. Moscow.

[29] Mironovskii B.N. 1965, Asronomicheskii Zhurnal, XLII, n.3, 977 (in Russian)

[30] Nakamura T., Sasaki M., Tanaka T, Thorne K.S. 1997, astro- ph/9708060 v1, 07 Aug.

[31] Novikov I.D., Frolov V.P. 1998. Black hole physics: basic consepts and new developements. Kluwer. Dordrecht.

[32] Novikov I.D., Polnarev A.G., Starobinsky A.A., Zeldovich Ya.B. 1979, Astron. Ap., 80, 104.

[33] Naselskij P.D., Novikov D.I,, Novikov I.D. 2003. Relict Radiation in the Universe. Nauka. Moscow.

[34] Page D.N. 1976, Phys. Rev.D 13, 198.

[35] Peebles P.J.E. 1971. Physical Cosmology. Princeton Univ. Press.

[36] Pisarev A.F., 1979, Preprint JINR 1979 NP13-12845 (in Russian)

[37] RudenkoV.N. 2003, gr-qc/0307105. 24 Jul.

[38] Rudenko V.N.,Sazhin M.V., 1980, Kvantovaya Electronika, v.7,(11),2344; translated as: Sov.Quantum Electron. 10,(11),1366.

[39] Servin M, Brodin G. 2003, gr-qc/0302039 27 Aug.

[40] Shoemaker D. et.al gr-qc/0308043 $14 \mathrm{Aug}$

[41] Weinberg S. Gravitation and Cosmology, 1972, ed. J.Wiley \& Sons Inc, NY.

[42] Zeldovich Y.B. 1973, Sov. Phys. JETP, 65, 1311.

[43] Zeldovich Ya.B.,1980, in General relativity, ed. Hawking S., Israel, Cambridge Univ.Press. (translation in Russian, p.217, ed. Mir 1983)

[44] Zeldovich Ya.B., Sunyaev R.A. 1970, Ap. Space Sci. 7, 20. 